J. RADIAT. RES., SUPPLEMENT, 162-167 (1991)

A Review of Forty-Five Years Study of Hiroshima and Nagasaki Atomic Bomb Survivors

II. BIOLOGICAL EFFECTS

\author{
Atomic Bomb and Leukemia \\ MICHITO ICHIMARU, MASAO TOMONAGA, TATSUHIKO AMENOMORI AND \\ TATSUKI MATSUO
}

\author{
Department of Hematology, Atomic Disease Institute, \\ Nagasaki University School of Medicine, Nagasaki, Japan
}

\begin{abstract}
Atomic bomb/Leukemia/Threshold/FAB classification/Chromosome
Characteristic features of leukemia among atomic bomb survivors were studied. The ratio of a single leukemia type to all leukemias was highest for CML in Hiroshima, and the occurrence of CML was thought to be most characteristic for atomic bomb radiation induced leukemia. In the distribution of AML subtypes of FAB classification, there was no $\mathrm{M} 3$ cases in 1Gy or more group, although several atypical AML cases of survivors were observed. Chromosome study was conducted using colony forming cells induced by hemopoietic stem cells of peripheral blood of proximal survivors. Same chromosome aberrations were observed in colony forming cells and peripheral blood of proximal survivors.
\end{abstract}

\title{
INTRODUCTION
}

The leukemias that occurred among atomic bomb survivors are one of the important model of the radiation induced human leukemia. There are some differences among the atomic bomb induced leukemia, so-called secondary leukemia and de novo leukemia. We shall describe some characteristic features of the leukemias which were probably induced by atomic bomb radiation. The effect of atomic bomb radiation on human body was mainly due to simple whole body irradiation.

The bone marrow of proximally exposed survivors became aplastic by heavy dose of radiation, however, the reproduction of new blood cells in the bone marrow was already seen in proximally exposed persons who died about 2 months after the bomb. The leukemia occurred among those exposed persons whose blood cells had recovered to a normal count after the bomb.

Leukemia cases among atomic bomb survivors increased gradually from 1946, and the peak of occurrence was observed about 6 years after the bomb ${ }^{11}$.

Fig. 1 shows the number of cases and types of leukemia in Hiroshima and Nagasaki among those who received $0.01 \mathrm{~Gy}$ or more.

There are some differences in leukemia type between the two cities. The ratio of chronic myelogenous leukemia $(\mathrm{CML})$ to all leukemia is significantly higher in Hiroshima than in Nagasaki. The risk of all types of leukemia increases with dose in both cities, except for Nagasaki survivors who received less than $1 \mathrm{~Gy}$ Kerma total dose (average marrow dose). This difference between two cities is clearly observed in CML incidence, but is not so clear in acute leukemia. Accordingly, this difference seems to be due to the difference in CML incidence between the two cities. The 




Fig. 1. Cases and type of leukemia among atomic bomb survivors who received $0.01 G y$ or more (1945-1975).

risk of CML in Hiroshima increases from individuals who received less than $0.5 \mathrm{~Gy}$ Kerma total dose, but no increase in Nagasaki in this dose. Another important point of atomic bomb induced leukemia is that leukemogenic risk has been higher among the survivors who were younger at atomic bomb in earlier stage. This tendency is most clear in CML cases ${ }^{2)}$.

The apparent threshold of acute leukemia and CML was reviewed using the leukemia samples of Hiroshima.

The features of CML in Hiroshima are most characteristic to atomic bomb radiation induced leukemia. This increased incidence of CML is much different from so-called secondary leukemia ${ }^{3)}$.

The peak of the occurrence of CML was observed 6 years after the bomb also in the below $1 \mathrm{~Gy}$ group as in the $1 \mathrm{~Gy}$ or more group. Accordingly, there might be radiation effect to induce CML occurrence in the below 1 Gy group also. The same phenomenon of CML was observed in three groups of 0.5 or more, $0.1 \sim 0.49$ and below 0.09 Gy (T65D dosimetry).

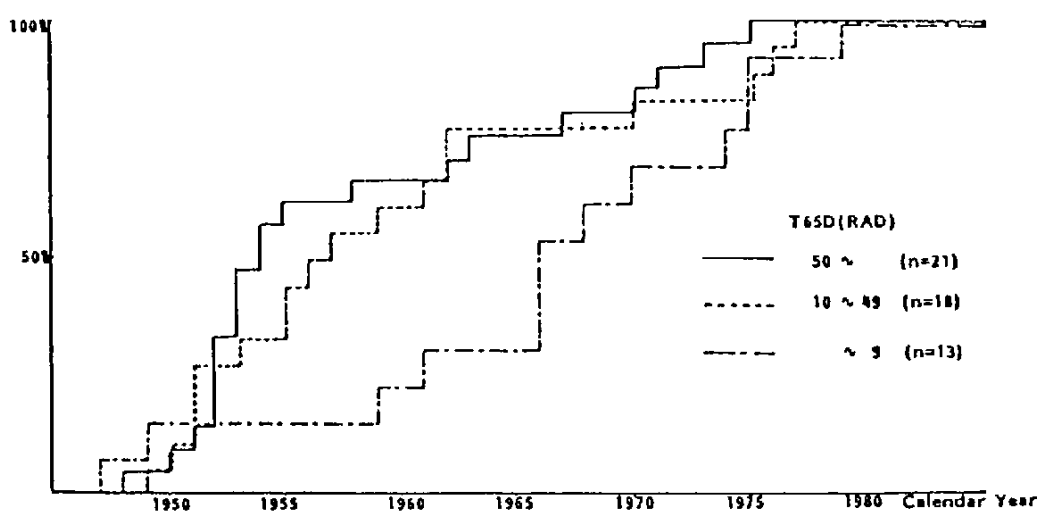

Fig. 2. Cumulative Cases of Chronic Myelogenous Leukemia by Radiation Dose. 


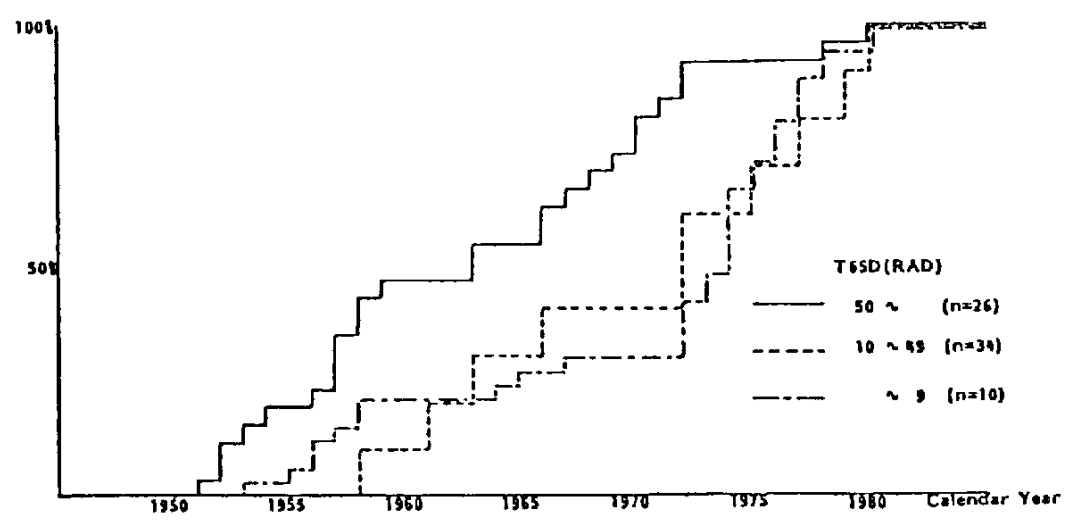

Fig. 3. Cumulative Cases of Acute Myeloid Leukemia by Radiation Dose.

Fig. 2 shows the percentage of cumulative CML cases in each year from 1946 to 1980 .

The shape of occurrence curve is the same in the $0.5 \mathrm{~Gy}$ or more and $0.1 \sim 0.49 \mathrm{~Gy}$ groups, but different in the below $0.09 \mathrm{~Gy}$ group. Therefore, it is suggested that an apparent threshold of CML occurrence is between 0.5 and $0.09 \mathrm{~Gy}$.

On the other hand, the shape of acute leukemia in the $0.1 \sim 0.49 \mathrm{~Gy}$ group was similar to that in the below 0.09 Gy group as shown in Fig. 3 .

When acute leukemia cases in Hiroshima are divided into two groups by exposure dose (1Gy or more and below 1Gy), there is no peak of occurrence unlike CML in the lower dose group. These findings suggest that the apparent threshold of acute leukemia occurrence may be near $1 \mathrm{~Gy}$.

Therefore, it was suspected that the characteristic feature of $\mathrm{A}$-bomb induced acute leukemia might be in the cases who received 1 Gy or more.

Recently, blood samples of leukemia cases among atomic bomb survivors have been reviewed by FAB (French-American-British) classification. The review has been completed for about $65 \%$ of the cases ${ }^{5)}$.

As shown in Table 1, ALL and MDS increased compared with the control group in 1Gy or more group. CML increased in $1 \mathrm{~Gy}$ or more and $0.01 \sim 0.99 \mathrm{~Gy}$ groups. In the distribution

Table 1. Leukemia Types by FAB Classification and Radiation Dose (both cities combined)

\begin{tabular}{lrrr}
\hline & & \multicolumn{1}{l}{ T65D } \\
& $0 \mathrm{~Gy}$ & $0.01-0.99 \mathrm{~Gy}$ & $1 \mathrm{~Gy}$ \\
\hline AML & $120(65.9 \%)$ & $49(45.8 \%)$ & $33(31.7 \%)$ \\
ALL & $20(11.0 \%)$ & $15(14.0 \%)$ & $27(26.0 \%)$ \\
MDS & $10(5.5 \%)$ & $4(3.7 \%)$ & $13(12.5 \%)$ \\
CML & $32(17.6 \%)$ & $39(36.5 \%)$ & $31(29.8 \%)$ \\
\hline Total & $132(100 \%)$ & $107(100 \%)$ & $104(100 \%)$
\end{tabular}


Table 2. Distribution of AML Subtypes by FAB Classification (Atomic Bomb Survivors)

\begin{tabular}{lccc}
\hline & & T65D & \\
& 0 Gy & $0.01-0.99$ Gy & 1 Gy \\
\hline M1 & 33 & 11 & 12 \\
M2 & 30 & 12 & 7 \\
M3 & 16 & 9 & 0 \\
M4 & 20 & 8 & 6 \\
M5 & 10 & 1 & 1 \\
M6 & 8 & 2 & 1 \\
\hline Total & 117 & 43 & 27
\end{tabular}

by age at bomb, the ratio of AML and ALL of below 15 years of age was largest. In the distribution of AML subtypes, there was no M3 case in the 1Gy or more group. (Tab. 2)

In general, there is no specific difference between AML of the survivors and de novo AML.

However, we find some non typical and interesting cases in survivors' acute leukemia. We would like to describe some of those cases.

The 1st case was diagnosed as aplastic anemia six years after the bomb (estimated dose was 3.81Gy). Severe pancytopenia and general hypoplastic marrow, but partial blast cell proliferation was found in marrow which suggests an existence of leukemia.

The 2 nd case suffered from severe progressive anemia in 1952, and died 3 months after. Estimated dose was 2.79Gy. Autopsy revealed diffuse bone marrow fibrosis and immature cell infiltration in the liver and kidney.

The 3rd case was admitted because of malaise and fever 20 years after the bomb. Lymphonode swellings and hepatosplenomegaly were noticed. Bone marrow was dry tap. The estimated radiation dose was $1.63 \mathrm{~Gy}$. The patient died after 6 months. The autopsy diagnosis was acute leukemia and malignant myeloskerosis. Immature cell infiltration was observed in the liver and kidney.

The 4th case developed acute leukemia with hepatosplenomegaly 16 years after the bomb. The estimated dose was $1.29 \mathrm{~Gy}$ and the patient had severe anemia and thrombocytopenia. The

Table 3. 5 Cases of Atypical Leukemia and Their Peripheral Blood

\begin{tabular}{lcccccc}
\hline & & & & & & \\
\cline { 5 - 7 } Case & Age, (age ATB) & $\begin{array}{c}\text { Dose } \\
(\mathrm{Gy})\end{array}$ & $\begin{array}{l}\text { RBC } \\
\times 10^{4}\end{array}$ & WBC & $\begin{array}{l}\text { Plate } \\
\times 10^{4}\end{array}$ & $\begin{array}{l}\text { Blast } \\
(\%)\end{array}$ \\
\hline 1. K.K. & $51(45)$ & 3.81 & 54 & 3200 & 1.2 & 0 \\
2. C.M. & $22(15)$ & 2.79 & 77 & 3900 & 1.3 & 5 \\
3. M.I. & $39(19)$ & 1.63 & 270 & 3000 & & 1 \\
4. Y.I. & $31(14)$ & 1.29 & 201 & 9700 & 8.0 & 3 \\
5. M.H. & $59(39)$ & 2.49 & 91 & 5200 & 9.6 & 5 \\
\hline
\end{tabular}


appearance of erythroblasts and megakaryocytes with myeloblasts were observed in peripheral blood.

Abnormal immature cell proliferation of three blood series was observed in the bone marrow. The diagnosis was acute panmyelosis.

The 5 th case was diagnosed to have acute myelogenous leukemia 20 years after the bomb with an estimated radiation dose was $2.49 \mathrm{~Gy}$. Abnormal proliferation of three blood series was also recognized in the bone-marrow. (Tab. 3)

As stated above, the increase of CML, the feature of leukemia types by FAB classification, and the cases of acute leukemia with abnormal findings in three blood series suggest that the initial leukemogenic effect of the atomic bomb radiation occurred in hemopoietic stem cells.

\section{CHROMOSOME STUDY}

Finally, we would like to describe the chromosome aberrations in atomic bomb survivors.

Even now, significantly increased chromosome aberrations due to atomic bomb radiation are recognized in peripheral blood $\mathrm{T}$-cells ${ }^{6}$ and bone marrow cells in proximally exposed atomic bomb survivors. Chromosome study was performed using colony forming cells induced by hemopoietic stem cells of peripheral blood of proximally exposed survivors whose chromosome aberrations were confirmed in peripheral blood T-cells ${ }^{7)}$. The same chromosome aberrations in colony forming cells and peripheral T-cells were observed in several survivors (Fig. 4).

This also suggests that atomic bomb radiation affected hemopoietic stem cells and these cells differentiated to peripheral T-cells.

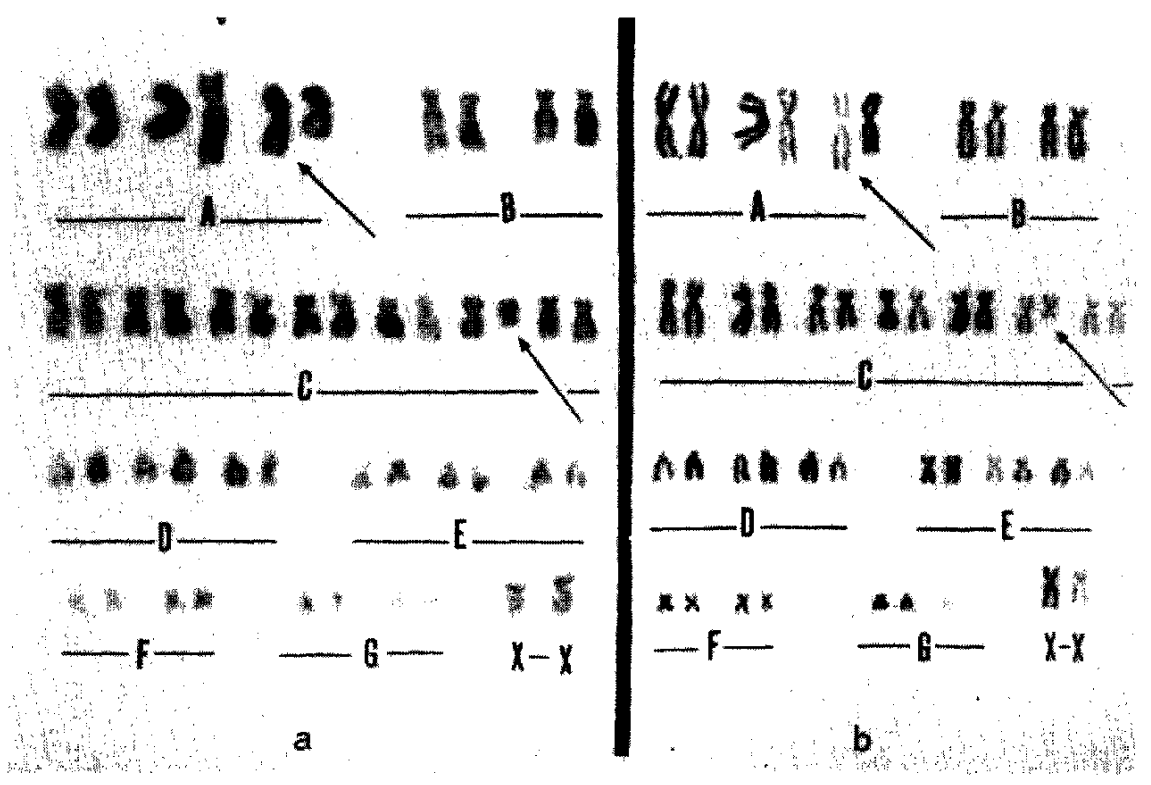

Fig. 4. Abnormal karyotypes $46, \mathrm{XX}, \mathrm{t}(3 \mathrm{q}+; \mathrm{Cq}-)$ seen in (a) a circulating burst and (b) peripheral T-lymphocyte of an atomic bomb survivor. They closely resemble each other. 


\section{CONCLUSION}

1) There were some qualitative differences in leukemia type among atomic bomb survivors between Hiroshima and Nagasaki.

2) The risk of chronic myelogenous leukemia was higher in Hiroshima than in Nagasaki. This increase of CML may be the different feature of atomic bomb survivors' leukemia from the so-called secondary leukemia.

3) There was no remarkable difference in feature between the leukemia of atomic bomb survivors and de novo leukemia, although several non-typical cases have been observed.

4) In 1 Gy or more group, ALL and MDS increased compared to the control group, and there was no $\mathrm{M} 3$ case in the distribution of AML subtypes (FAB classification).

5) The apparent threshold of CML occurrence in Hiroshima might be between $0.5 \sim 0.09 \mathrm{~Gy}$, and that of acute leukemia near $1 \mathrm{~Gy}$.

6) Same chromosome aberrations were observed in both colony forming cells induced from stem cells as well as in the peripheral T-cells suggesting that the initial leukemogenic effects of atomic bomb radiation was in hemopoietic stem cells.

The present report is based upon T65D estimated doses ${ }^{8)}$.

\section{ACKNOWLEDGMENTS}

This leukemia study of atomic bomb survivors has been done as a cooperative study by Nagasaki University School of Medicine and Hiroshima University School of Medicine and Radiation Effects Research Foundation.

\section{REFERENCES}

1. Ichimaru, M., Ohkita, T. and Ishimaru, T. (1986) Leukemia, multiple myeloma and malignant lymphoma. Gann Monograph on Cancer Research 32: 113.

2. Ichimaru, M., Ishimaru, T. and Belsky, J.L. (1978) Incidence of leukemia in atomic bomb survivors belonging to a fixed cohort in Hiroshima and Nagasaki, 1950-71. Radiation dose, years after exposure, age at exposure and type of leukemia. J. Radiat. Res. 19: 262.

3. Moloney, W.C. (1987) Radiogenic leukemia revised. Blood 70: 905.

4. Bennett, J.M., Catovsky, D., Daniel, M.T., Flandrin, G., Galton, D.A.G., Gralnick, H.R. and Sultan, C. (1976) Proposals for the classification of the acute leukemia. Br. J. Haemat. 33: 451.

5. Matsuo, T., Tomonaga, M., Bennett, J.M., Kuriyama, K., Imanaka, E., Kuramoto, A., Kamada, N., Ichimaru, M., Finch, S.C., Piciotta, A.V. and Ishimaru, T. (1988) Reclassification of leukemia among A-bomb survivors in Nagasaki, using FAB classification for acute leukemia. Jpn. J. Clin. Oncol. 18: 91.

6. Awa, A.A., Sofuni, T., Honda, T., Hoh, M., Neriishi, S. and Otake, M. (1978) Relationship between radiation dose and chromosome aberrations in atomic bomb survivors of Hiroshima and Nagasaki. J. Radiat. Res. 19: 126.

7. Amenomori, T., Tomonaga, M., Matsuo, T., Yoshida, Y., Kuriyama, K., Sadamori, N. and Ichimaru, M. (1988) A micromethod for chromosome preparation from individual hemopoietic colonies cultured in methycellulose. Int. J. Cell Cloning 3: 133.

8. Milton, R.C. and Shohoji, T. (1968) Tentative 1965 radiation dose estimation for atomic bomb survivors Hiroshima and Nagasaki. ABCC Tech. Rep. 1:68. 